\title{
Evidence for two competing order parameters in underdoped cuprates superconductors from a model analysis of the Fermi-arc effects
}

\author{
Andrés Greco \\ Facultad de Ciencias Exactas, Ingeniería y Agrimensura and Instituto de \\ Física Rosario (UNR-CONICET). Av. Pellegrini 250-2000 Rosario-Argentina.
}

(Dated: August 22, 2018)

\begin{abstract}
Preformed pairs above $T_{c}$ and the two-gap scenarios are two main proposals for describing the low doping pseudogap phase of high- $T_{c}$ cuprates. Very recent angle-resolved photoemission experiments have shown features which were interpreted as evidence for preformed pairs. Here it is shown that those results can be explained also in the context of the two-gap scenario if self-energy effects are considered. The discussion is based on the $d$-CDW theory or the flux phase of the $t-J$ model.
\end{abstract}

PACS numbers: 74.72.-h,71.10.Fd,71.27.+a

The low doping pseudogap (PG) phase of high- $T_{c}$ cuprates is one of the most relevant and controversial puzzles in condensed matter physics. $\stackrel{1}{=}$ Two main scenarios are disputing the explanation of the PG phase. In one of the scenarios the PG is associated to preformed pairs existing above $T_{c}$, while in the other, the so called two-gap scenario, the superconducting gap and the PG have different origins and may compete each other $\stackrel{2}{=}$ Whereas some experiments provide support for preformed pairs $3,4,5,6$ others are interpreted in terms of two competing phases $7,8,9,10,11,12,13,14$ One of the most unexpected and surprising results in the PG phase arises from angle-resolved photoemission spectroscopy (ARPES) which show that the Fermi surface (FS) is broken up into disconnected arcs,$\frac{1,15,16}{1}$ Very recent ARPES experiments which were interpreted in terms of preformed pairs $\frac{17,18,19}{17}$ brought new insights to this discussion. Interestingly, in Ref. 19] it was found that in the PG phase electronic excitations near the anti-node disperse like in a superconductor. Then, it is crucial to evaluate to what extent ARPES experiments may be compatible with the two-gap scenario. A well known theory for two competing orders is the $d$-CDW theory ${ }^{20}$ which proposes, phenomenologically, a normal state complex order parameter with $d$-wave symmetry (see Ref.[21] for a related proposal). Interestingly, at mean field level the $t-J$ model exhibits, at low doping and below a characteristic temperature $T_{F P}$, the flux phase (FP) which possesses the main properties to be identified with the $d$ CDW $\underline{22,23,24,25}$ In this paper it will be shown that some ARPES features can be understood after considering, beyond mean field, self-energy effects showing that a theory for two competing orders is compatible with the experiments.

Before presenting results beyond mean field level, the physical picture emerging from the mean field solution of the $t-J$ model is given for completeness. The mean field treatment of the $t-J$ model yields a quasi-particle (QP) dispersion $\epsilon_{k}=-2(t \delta+$ $r J)\left(\cos \left(k_{x}\right)+\cos \left(k_{y}\right)\right)-4 t^{\prime} \delta \cos \left(k_{x}\right) \cos \left(k_{y}\right)-\mu$, with $r=1 / N_{s} \sum_{\mathbf{k}} \cos \left(k_{x}\right) n_{F}\left(\epsilon_{k}\right) \stackrel{26}{2} n_{F}$ is the Fermi function, $\delta$ the doping away from half-filling, $\mu$ the chemical potential and $N_{s}$ the number of sites. $t, t^{\prime}$ and $J$ are

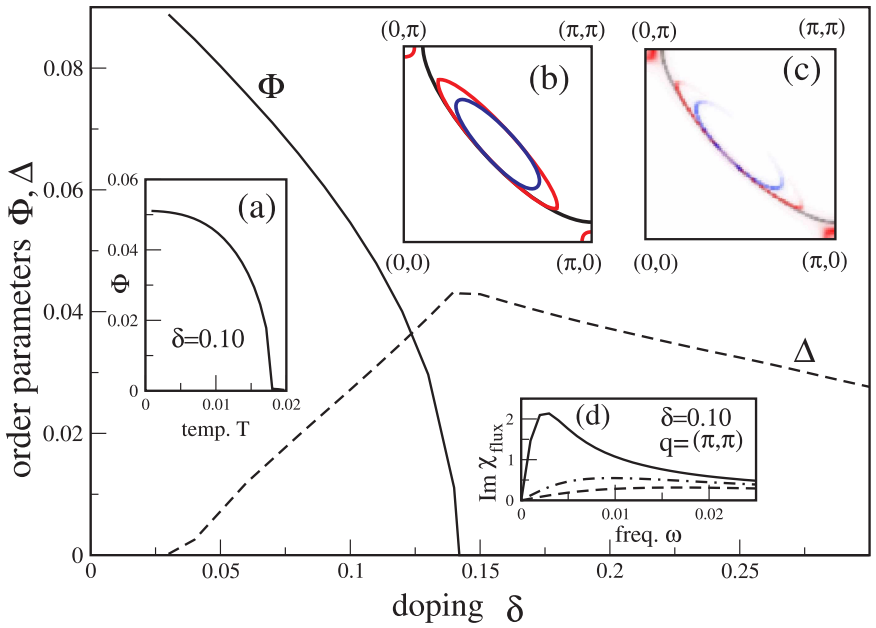

FIG. 1: (color online) Doping dependence of the superconducting $(\Delta)$ and $d$-CDW $(\Phi)$ order parameters at $T=0$. Inset (a) Temperature dependence of the $d$-CDW order parameter $\Phi$ for $\delta=0.10$. Inset (b) Evolution of the FS with decreasing temperature. Black line is the FS for $T>T_{F P}$, red line for $T=0.017(\Phi \sim 0.01)$ and blue line for $T=0.01(\Phi \sim 0.05)$. Inset (c) Spectral weight intensity on the FS for the same temperatures than inset (b), showing that the intensity of the outer part of the pockets is low. Inset (d) Softening of the $d$-wave flux mode approaching $T_{F P}$. Im $\chi_{\text {flux }}$ for $T=0.030$ (dashed line), $T=0.025$ (dot-dashed line) and $T=0.023$ (solid line).

hopping between nearest neighbor, next nearest neighbor and the nearest neighbor Heisenberg copling respectively. At low doping, the homogeneous mean field solution becomes unstable against a $\mathrm{FP}$ or $d$-CDW with order parameter $\Phi(k)=\Phi \gamma(k)$ which has $d$-wave symmetry like the superconducting gap $\Delta(k)=\Delta \gamma(k)$ being $\gamma(k)=1 / 2\left(\cos \left(k_{x}\right)-\cos \left(k_{y}\right)\right)$. Fig. 1 shows the doping dependence of $\Phi$ and $\Delta$ for $t^{\prime} / t=0.35$ and $J=0.3$ at zero temperature. In the following the lattice constant $a$ of the square lattice is used as length unit and $t$ as energy unit. In the overdoped (OD) region, $\delta \geqslant \delta_{c} \sim 0.14, \Phi$ is zero while in the underdoped (UD) region, $\delta \leqslant \delta_{c}, \Phi$ 
is nonzero and coexists with $\Delta$. The two order parameters compete each other leading to the decay of $\Delta$ with underdoping. ${ }^{25,27}$ Inset (a) shows the temperature dependence of $\Phi$ for $\delta=0.10$ discarding superconductivity, the instability occurs when the flux susceptibility ${ }^{28}\left(\chi_{\text {flux }}\right)$ diverges at $T_{F P} \sim 0.018$ for momentum $q \sim(\pi, \pi)$. $\chi_{f l u x}(\mathbf{q}, \omega)=\left(\frac{\delta}{2}\right)^{2}\left[(8 / J) r^{2}-\Pi(\mathbf{q}, \omega)\right]^{-1}$ where $\Pi(\mathbf{q}, \omega)$ is the electronic polarizability calculated with a form factor $\gamma(\mathbf{q}, \mathbf{k})=2 r\left(\sin \left(k_{x}-q_{x} / 2\right)-\sin \left(k_{y}-q_{y} / 2\right)\right)$; note that for $\mathbf{q} \sim(\pi, \pi)$ the form factor $\gamma(\mathbf{q}, \mathbf{k})$ transforms into $\sim\left(\cos \left(k_{x}\right)-\cos \left(k_{y}\right)\right)$ which indicates the $d$-wave character of the FP. From the dynamical point of view, with decreasing temperature from above, a $d$-wave flux mode with momentum $q=(\pi, \pi)$ softens, increases its spectral weight and reaches $\omega=0$ at $T_{F P}$ (inset (d)). Below $T_{F P}$ the translational symmetry is broken. Inset (b) shows the evolution of the Fermi surface with decreasing temperature. In the normal state above $T_{F P}$ the FS is large and $(\pi, \pi)$-centered. Below $T_{F P}, \Phi$ opens near $(\pi, 0)$ and the new FS consists of four small hole pockets near nodal direction. With decreasing $T, \Phi$ shows a fast increasing (inset (a)) and the side of hole pockets is strongly diminished. However, the spectral weight intensity of the outer part of the pocket is low $\stackrel{29}{,}$ resembling the presence of arcs (inset (c)). Although the mean field FP scenario shows features (phase diagram, two competing gaps, pockets, arcs, and other ones $26,27,30$ ) which may be assimilated with those observed in cuprates, there are drawbacks to be addressed. While the FP scenario predicts a true phase transition at $T_{F P}$, some experiments show a smooth crossover $17,18,31$ In addition, FP predicts well defined QP peaks above and below $T_{F P}$ everywhere on the Brillouin zone (BZ) while ARPES shows coherent peaks only near nodal direction. 16,32 Near the anti-node the QP peak is always broad, $\stackrel{16,32}{2}$ presenting a PG which seems to be filling up but not closing with increasing $T^{17,18,33}$ giving the impression of a smooth crossover of the spectral properties. In what follows it will be shown that this controversy can be solved after including selfenergy effects.

For discussing self-energy effects a large- $N(N$ is the number of spin components) approach is useful ${ }^{34}$ since it is possible to identify the most relevant fluctuations above mean field. As discussed in Ref. 28] the dominant contribution at low doping and low energy (thus a candidate for describing the $\mathrm{PG}$ ) is given by the coupling between quasi-particles and FP fluctuations. The $\mathcal{O}(1 / N)$ scattering rate reads

$$
\begin{gathered}
\operatorname{Im} \Sigma(\mathbf{k}, \omega)=-\frac{1}{N_{s}} \sum_{\mathbf{q}} \gamma^{2}(\mathbf{q}, \mathbf{k}) \operatorname{Im} \chi_{f l u x}\left(\mathbf{q}, \omega-\epsilon_{k-q}\right) \\
\times\left[n_{F}\left(-\epsilon_{k-q}\right)+n_{B}\left(\omega-\epsilon_{k-q}\right)\right]
\end{gathered}
$$

where $n_{B}$ is the Bose factor. Using the self-energy $\Sigma(\mathbf{k}, \omega)$ the spectral function $A(\mathbf{k}, \omega)$ is calculated as usual. Note that in $\mathcal{O}(1 / N)$ the mean field transition temperature $T_{F P}$ is not renormalized by fluctuations. Finally, it is important to point out that present calculation
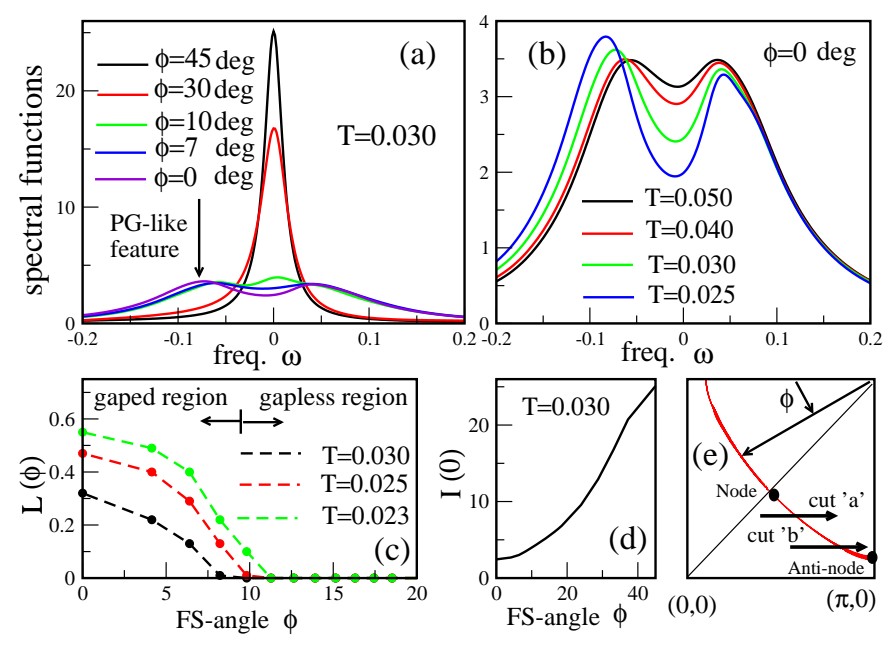

FIG. 2: (color online) (a) Spectral functions at $T=0.030$ for several Fermi momenta from the node $(\phi=45 \mathrm{deg})$ to the anti-node $(\phi=0 \mathrm{deg})$ for $\delta=0.10$. (b) Spectral functions at the anti-node for several temperatures for $\delta=0.10$. c) Loss of the low-energy spectral weight $L(\phi)$ along the FS for different temperatures and $\delta=0.10$. The length of the gaped (gapless) region decreases (increases) with increasing $T$. (d) Intensity of the spectral functions at $\omega=0(I(0))$ along the FS for $T=0.030$ and $\delta=0.10$. (e) Definition of the FS-angle $\phi$. The nodal and anti-nodal Fermi momenta are indicated. The arrows show cut 'a' and cut 'b' along which the spectral functions of Fig. 4 were calculated. $\omega$ is measured with respect to the Fermi energy. A broadening $\eta=0.01$ was used in the calculation of the spectral functions.

is also a way for discussing the role of self-energy corrections in the phenomenological $d$-CDW theory.

Present theoretical results will be presented in the standard form for an easier comparison with ARPES experiments. Fig. 2(a) shows $A(\mathbf{k}, \omega)$ for $\delta=0.10$ (Ref. 35]) and $T=0.030>T_{F P}$ for different momenta, on the normal state FS, labeled by the FS-angle $\phi$ (see Fig. 2(e)). A gapless spectrum with maximun at $\omega=0$ is observed near the node $(\phi=45 \mathrm{deg})$. Moving towards the anti-node $(\phi=0)$ the spectrum become broad, losses intensity at $\omega=0$ and develops a PG-like feature, suggesting the presence of arcs. Decreasing temperature approaching $T_{F P}$ the gaped region near the anti-node expands, e.i., the length of the arc decreases. Note that the arcs developed at $T>T_{F P}$ do not imply a breaking of the translational symmetry. The behavior of the intensity at $\omega=0(I(0))$ along the FS (Fig. 2(d)) indicates also the loss of QP coherence near the anti-node (see Fig. 2(b) in Ref. 36 for comparison with experiment). These PG-like features occur above but near $T_{F P}$ and are associated to self-energy effects proving the proximity to the FP instability via the coupling between quasi-particles and the $d$-wave soft flux modes. As discussed above, the soft mode has momentum $q \sim(\pi, \pi)$ allowing the scattering between the quasi-particles near the anti-nodes. Adopting the accepted value $t=0.4 \mathrm{eV}$ the temperature 

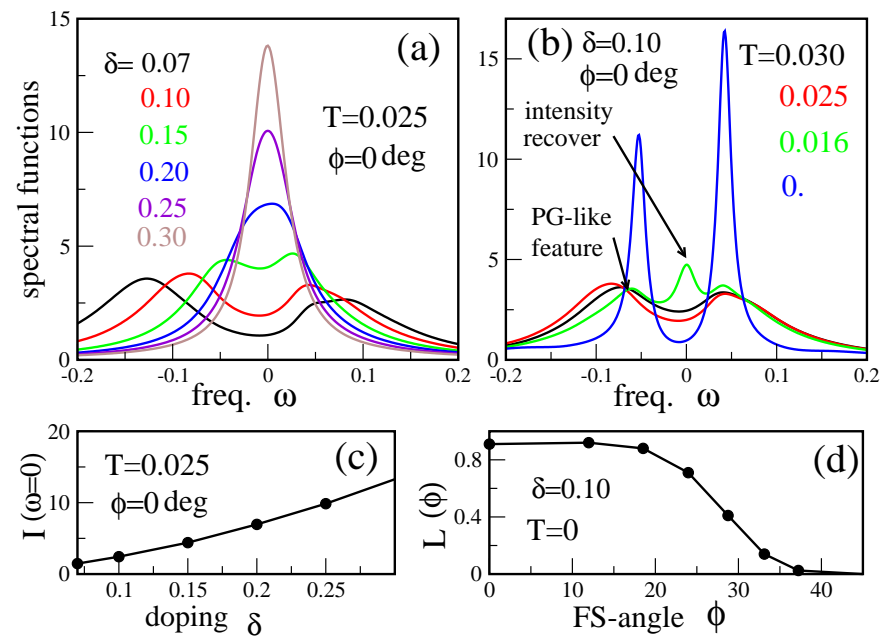

FIG. 3: (color online) (a) Spectral functions for $T=0.025$ at the anti-node for several dopings. (b) Spectral functions at the anti-node for $\delta=0.10$. For $T=0.030$ and $T=0.025$ the broad PG-like features are due to self-energy effects while for $T=0$ the true gap $\Phi$ dominates and the peak at the PG becomes sharp. In the transitional region at $T=0.016 \lesssim T_{F P}$ the broad PG-like feature at $\omega \sim-0.07$, due to self-energy effects, remains and a recover of the intensity at $\omega=0$ is observed. (c) Intensity at $\omega=0$ as a function of doping at the anti-node for $T=0.025$. (d) Loss of the low-energy spectral weight at $T=0$ for $\delta=0.10$.

range and the PG have the same order of magnitude than those in the experiment.

For comparison with ARPES results $\frac{18}{18}$ Fig. 2(c) shows the loss of the low-energy spectral weight $(L(\phi)=1-$ $\left.I(0) / I\left(\Delta_{\phi}\right)\right)$ along the FS for different temperatures. $\left(I\left(\Delta_{\phi}\right)\right.$ is the intensity at the PG as exemplified by the arrow in Fig. 2(a)). In agreement with the experiment (see Fig. 3 of Ref. [18]) the arc length expands with increasing $T$. Note also that $L(\phi=0)$ decreases with increasing temperature. $\stackrel{18}{ }$ In addition, the temperature behavior of the spectral functions at the anti-node indicates that the PG-like feature is filling but not closing (Fig. 2(b)). This behavior leads to a PG rather temperature independent in qualitative agreement with Refs. [17, 18 33]. In the experiment of Ref. [18] this behavior seems to continue in the superconducting state, thus the existence of only one gap was assumed as favoring the interpretation of preformed pairs. It is worth to mention that on a two-gap scenario the explanation of this behavior is not straightforward (see for instance Ref.[37]). However, in several ARPES experiments $\frac{12,13,14}{1}$ two gaps are detected, making doubtful the interpretation of Ref. [18]. In spite of this discrepancy is presently unclear, in the present paper it is shown that some ARPES data can be described in the context of the two-gap scenario.

Next, the dependence with doping is discussed. Fig. 3 (a) shows the spectral functions at the anti-node for different dopings at $T=0.025$. With overdoping the
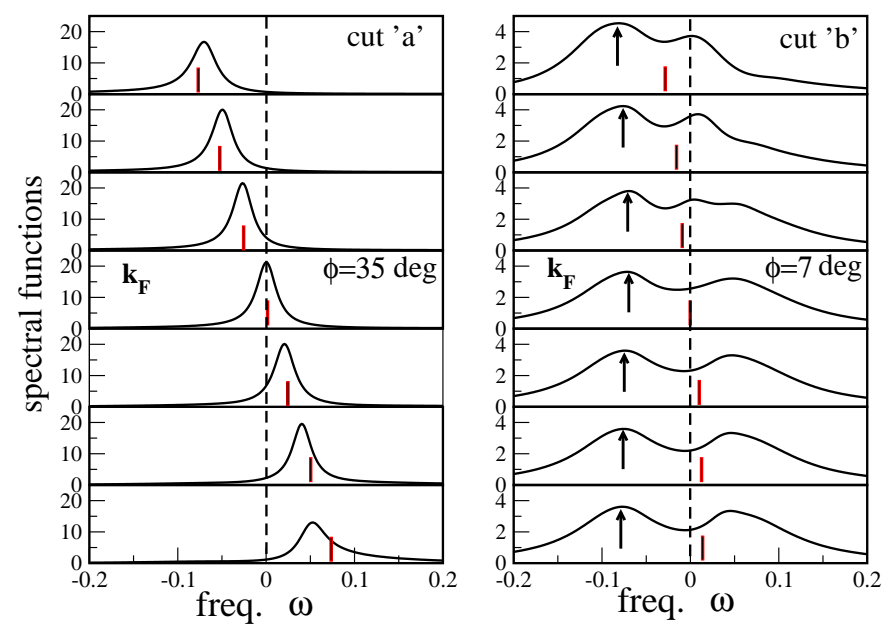

FIG. 4: (color online) Spectral functions for $\delta=0.10$ along the cut 'a' (left panel) and cut 'b' (right panel) at $T=0.025$. Cut 'a' (Cut 'b') passes trough the gapless (gaped) Fermi momentum at $\phi=35 \mathrm{deg}(\phi=7 \mathrm{deg})$. Vertical dashed line indicates the Fermi level. Red marks show the location of the unrenormalized QP peaks.

PG-like feature closes and, simultaneously, the intensity at $\omega=0$ increases (see also Fig. 3(c)), emerging the QP peak as in the experiment 32,38 Note that from present approach the PG-like features are expected, in the normal state, even for $\delta \gtrsim \delta_{c}$. This fact indicates that there is no contradiction between the existence of PG features in nearly overdoped samples $\frac{16}{}$ and theories, like $d$-CDW or FP, which suggest the existence of a quantum critical point near optimal doping.

In the following it will be shown that recent ARPES results 19 (see also Ref. [39]), interpreted as evidence for preformed pairs above $T_{c}$ due to the apparent Bogoliubov-like dispersion of quasiparticles near the antinode, are also consistent with a theory for two competing order parameters. Fig. 4 shows spectral functions for $\delta=0.10$ at $T=0.025$ along cut 'a' (left panel ) and cut 'b' (right panel) which are parallel to the $(0,0)-(\pi, 0)$ direction (Fig. 2(e)). As in Fig. 3 of Ref. [19] the peaks are better defined in the arc, or gapless region (cut 'a'), than in the gaped region (cut 'b') where only broad features are seen. While for cut 'a' the low energy peak approaches $k_{F}$, for cut 'b' the broad feature (marked with arrow in right panel) back bends slightly. According to present theory the reason for this behavior relays on a different fact to that discussed in Ref. [19]. Indeed, the broad feature along cut 'b' is due to self-energy effects coming from the interaction between quasi-particles and the soft modes in the proximity to the $d$-CDW or FP instability.

Now, results for $T<T_{F P}$ are discussed. For $T \ll T_{F P}$ a large gap $\Phi$ occurs in the electronic spectrum what affects $\chi_{\text {flux }}$ and washes the effect of the low energy $d$ CDW soft mode on the self-energy. In this case the ex- 
pected situation is close to that predicted for the pure $d$-CDW picture (see Fig. 3(b) for $T=0$ ) and pockets would be observed. ${ }^{40}$ On the other hand, the peak at the PG becomes much sharper than those observed at $T>T_{F P}$ as can be seen by comparing the result for $T=0$ with those for $T=0.030$ and $T=0.025$. In addition, at $T=0$, the loss of the low-energy spectral weight $L(\phi)$ (Fig. 3(d)) follows the typical behavior expected for a $d$-wave gap. There is a range of temperatures below but near $T_{F P}$ where $\Phi$ is small (Fig. 1(a)) and its value competes with the energy of the soft mode. In this region a recovery of the intensity at $\omega=0$ is observed together with the broad PG-like feature that appears at $T>T_{F P}$ (see Fig. 3(b) for $T=0.016$ ). Note that the range of temperatures which marks the transition from PG-like features due to self-energy effects for $T>T_{F P}$ towards the situation mainly dominated by the true gap
$\Phi$ for $T \ll T_{F P}$ is small. Searching in the above findings could contribute to the discussion about the dichotomy between: a) Fermi $\operatorname{arcs}^{17,18,41}$ and Fermi pockets, $, 42,43$ b) smooth crossover ${ }^{17,18,31}$ and abrupt transition $9,44,45$ and c) one gap ${ }^{18}$ and two gaps, $\stackrel{12,13}{ }$

In summary, it was shown that recent ARPES experiments do not necessarily imply the existence of preformed pairs above $T_{c}$. These experiments can also be described in the context of the two competing order parameters if self-energy effects are take into account in the proximity of the opening of a normal state gap like in the case of $d$ CDW or the flux phase. Finally, present paper supports theories invoking two competing gaps as the explanation for the low doping properties of cuprates.

The author thanks to M. Bejas, J.C. Campuzano, A. Foussats, A. Muramatsu, H. Parent, and R. Zeyher for valuable discussions.
1 T. Timusk and B. Statt, Rep. Prog. Phys. 62, 61 (1999).

2 S. Hüfner, M.A. Hossain, A. Damascelli, and G.A. Sawastzky, Rep. Prog. Phys. 71, 062501 (2008). M. R. Norman, D. Pines and C. Kallin, Adv. Phys. 54, 715 (2005).

${ }^{3}$ H. Alloul et al., Phys. Rev. Lett. 70, 1171 (1993).

${ }^{4}$ O. Fischer et al., Rev. Mod. Phys. 79, 353 (2007).

${ }^{5}$ Y. Wang et al., Phys. Rev. Lett. 95, 247001 (2005).

6 Y. Wang, L. Li and N. P. Ong, Phys. Rev. B 73, 024510 (2006).

7 M. Le Tacon et al., Nature Physics 2, 537 (2006).

${ }^{8} \mathrm{Li} \mathrm{Yu}$ et al., Phys. Rev. Lett. 100, 177004 (2008).

9 H.A. Mook et al., Phys. Rev. B 78, 020506(R) (2008).

10 Y.H. Liu et al., Phys. Rev. Lett. 101, 137003 (2008).

11 R. Khasanov et al., Phys. Rev. Lett. 101, 227002 (2008).

12 J.-H. Ma et al., Phys. Rev. Lett. 101, 207002 (2008).

13 T. Yoshida et al., Phys. Rev. Lett. 103, 037004 (2009).

14 T. Kondo et al., Nature 457, 296 (2009). T. Kondo et al., Phys. Rev. Lett. 98, 267004 (2007).

15 M.R. Norman et al., Nature 392, 157 (1998).

16 A. Damascelli, Z. Hussain, and Z.-X.Shen, Rev. Mod. Phys. 75, 473 (2003).

17 A. Kanigel et al., Nature Physics 2, 447 (2006).

18 A. Kanigel et al., Phys. Rev. Lett. 99, 157001 (2007).

19 A. Kanigel et al., Phys. Rev. Lett. 101, 137002 (2008).

20 S. Chakravary, R. B. Laughlin, D.K. Morr, and C. Nayak, Phys. Rev. B 63, 094503 (2001).

21 C.M. Varma, Phys. Rev. B 73, 155113 (2006).

${ }^{22}$ I. Affleck and J.B. Marston, Phys. Rev. B 37, 3774 (1988).

${ }^{23}$ G. Kotliar, Phys. Rev. B 37, 3664 (1988).

24 D.C. Morse and T.C. Lubensky, Phys. Rev. B 43, 10436 (1991).

25 E. Cappelluti and R. Zeyher, Phys. Rev. B 59, 6475 (1999).
26 A. Greco and R. Zeyher, Phys. Rev. B 70, 024518 (2004).

27 R. Zeyher and A. Greco, Phys. Rev. Lett. 89, 177004 (2002).

28 A. Greco, Phys. Rev. B 77, 092503 (2008).

29 S. Chakravary, C. Nayak, and S. Tewari, Phys. Rev. B 68, 100504 (2003).

30 S. Chakravary and H.-Y. Kee, Proc. Natl. Acad. Sci. USA 105, 8835 (2008).

31 J.L. Tallon and J.W. Loran, Physica C 349, 53 (2001).

32 C. Kim et al., Phys. Rev. Lett. 80, 4245 (1998).

33 M.R. Norman, M. Randeria, H. Ding, and J.C. Campuzano, Phys. Rev. B 57, 11093(R) (1998).

34 A. Foussats and A. Greco, Phys. Rev. B 70, 205123 (2004).

35 The doping value $\delta=0.10$ is similar to the corresponding doping of the underdoped samples studied in Refs. 17, 18, 19].

36 M.R. Norman et al., Phys. Rev. B 76, 174501 (2007).

37 R.S. Markiewicz, C. Kusko, and V. Kidambi, Phys. Rev. B 60, 627 (1999).

38 A. Kamiski et al., Phys. Rev. B 71, 014517 (2005).

39 M. Shi et al., arXiv:0810.0292

40 Since the present paper is focused in the pseudogap regime, superconductivity was discarded. Due to the fast increasing of $\Phi$ with decreasing $T$ results for $T=0$ are expected for $T>T_{c}$.

41 M.A. Hossain et al., Nat. Phys. 4, 527 (2008).

42 J. Chang et al., New Journal of Physics 10, 103016 (2008).

43 N. Doiron-Leyraud et al., Nature 447, 565 (2007).

44 B. Fauque et al., Phys. Rev. Lett. 96, 197001 (2006).

45 B. Leridon, P. Monod, and D. Colson, arXiv:0806.2128. 